\title{
Total intensity radio variations in blazars
}

\author{
Esko Valtaoja ${ }^{1}$, Markku Lainela, Elina Lindfors, Pia-Maria Saloranta, Kaj Wiik \\ Tuorla Observatory and Department of Physics, University of Turku \\ Väisäläntie 20, FI-21500 Piikkiö, Finland \\ E-mail: esko.valtaoja@utu.fi
}

\author{
Talvikki Hovatta, Anne Lähteenmäki, Elina Nieppola, Ilona Torniainen, Merja \\ Tornikoski \\ Metsähovi Radio Observatory, Helsinki Unversity of Technology \\ Metsähovintie 114, FI-02540 Kylmälä, Finland
}

\begin{abstract}
We discuss the uses of total flux density monitoring for studies of blazars. By its very nature, monitoring requires large amounts of dedicated telescope time. Such efforts are, however, necessary, since the synchrotron continuum radiation provides only very limited amounts of information, and a single-epoch multifrequency snapshot spectrum is hardly ever sufficient to constrain possible theories and models for the emitting regions. In contrast, analysis of dedicated multifrequency monitoring data obtained over years can reveal the nature of variations, the physics of the synchrotron-emitting regions (shocked jets), and also provide information relevant to other processes such as the gamma-ray emission. Monitoring can also be used to derive fundamental parameters of the jets, their speeds, luminosities and viewing angles, fundamental data in our efforts to understand how active galactic nuclei are powered by supermassive black holes.
\end{abstract}

Workshop on Blazar Variability across the Electromagnetic Spectrum

Palaiseau, France

April $22^{\text {nd }}-25^{\text {th }} 2008$

$1 \quad$ Speaker 


\section{Introduction}

By definition, blazars are sources in which the radiation is dominated by the synchrotron component (or, in the high-energy regime, by the inverse Compton component closely related to the synchrotron component). Although other components such as lines or thermal continuum may be detectable, or even dominant, in some frequency regimes, in the radio to near infrared frequencies only synchrotron radiation can be observed. This makes study of blazars both simple and difficult. With just one well understood emission mechanism, interpretation of data is much easier than, say, in the X-ray regime of ordinary AGN where even the nature of radiation is often unknown. On the other hand, synchrotron radiation carries notoriously little information.

The origin of the blazar synchrotron component is well established. It comes from the relativistic outflow - always just one outflow, since the two original outflows are respectively Doppler boosted and de-boosted - along the rotation axis of the supermassive black hole. Beyond this statement, little is known for certain. We do not know the composition of the outflow, its energy budget, whether is has a significant gas (proton) component, how it is energized, accelerated and collimated, at what distance from the supermassive black hole it originates, how it interacts with the environment, what is the internal structure of the flow, and so forth. We are rather certain that most, if not all, major flux variations are due to growing and decaying shocks in the jet, but again the physical details remain contested. With high likelihood, the inverse Compton component is created by the same relativistic electrons as the synchrotron component, but the site(s) of IC radiation and the nature of the seed photons are not known. Nature clearly produces a variety of AGN types with a wide range of jet parameters, but we have little clue how the various classes of sources with jets relate to each other, and how they are created by variations in the fundamental parameters such as the black hole mass, spin, and accretion rate. It is a sobering reminder that most of these questions (and others not listed here) were posed decades ago. Much remains to be done, and much of it can be done only with information squeezed out of the total intensity variations of the synchrotron component - since we have only them and VLBI observations to study the relativistic outflows.

Total flux density monitoring is a very laborious undertaking. The close to 100000 observations at mainly $22 \mathrm{GHz}$ and $37 \mathrm{GHz}$, obtained with the Metsähovi radio telescope in Finland, have required a comparable number of telescope and data reduction hours during the almost 30 years of AGN monitoring. In this paper we describe some past and future uses of the data obtained in the Metsähovi monitoring program, and in other radio and optical monitoring programs. We try to show that such huge, decades-spanning monitoring efforts are absolutely necessary, if we ever wish to understand blazars and relativistic jets in general. Although much has been achieved, we hope to demonstrate that the best is yet to come regarding answers to the questions posed above.

This paper is divided into three main sections reflecting three of the main research directions presently pursued in the AGN monitoring program with the Metsähovi 14-m radio telescope, a joint effort between the Metsähovi Radio Observatory of the Helsinki University of Technology and the Tuorla Observatory of Turku University. What is the nature of the total flux 
density variations - what causes the synchrotron flux and its variations? What is the importance of multifrequency monitoring - what is the advantage of a movie over a snapshot? How to study the fundamental physics of AGN - what are the basic physics of relativistic jet flows?

\section{Nature of total flux density variations}

Although a large number of theoretical papers have been devoted to the physics of relativistic outflows in AGN - in other words, to the physics of blazars, although many other AGN also have less dominant jets - two publications stand out as the cornerstones of our present theoretical understanding. Almost thirty years ago, Roger Blandford and Arieh Königl published their seminal paper, Relativistic Jets as Compact Radio Sources, which set the basics of outflow physics and their relation to observations, both total flux and VLBI data [1]. In the words of the abstract: "It is proposed that radio emission originates both from the quasi-steady jet itself and from behind strong shock waves which either propagate in the jet, or which are formed behind dense concentrations (clouds) that are accelerated to relativistic speeds by the flow."

The physics of the "strong shock waves" were elucidated six years later by Alan Marscher and Walter Gear in the paper Models for High-Frequency Radio Outbursts in Extragalactic Sources, which described the still-current framework for flux variations [2]. Investigating a well-observed flare in 3C 273, they concluded that the observations were best explained by a "model in which the outburst is due to a shock wave passing through an adiabatic, conical, relativistic jet" and formulated a simple mathematical model for the shock.

These two papers provided a clear, simple framework within which observations could be compared with theory and the parameters of the underlying jet and the shocks could be derived from data obtained by total flux density and VLBI observations. Although they definitely are "cartoon models", from an observer's point of view they remain extremely useful, since the very limited amount of obtainable data usually cannot really be quantitavely compared with, say, hydrodynamical simulations or more complicated theoretical models with a large number of essentially free parameters.

\subsection{Testing shocked jet models}

Using the Metsähovi monitoring data from the first five years of observations, we made the first extensive tests of the Marscher \& Gear model a few years after the publication of the original paper $[3,4]$. The $M \& G$ framework provides preditions of the spectral shape and its changes during the evolution of a radio flare, as well as of its temporal evolution in the fluxfrequency space, characterised by the motion of the synchrotron spectral peak $S_{\max }\left(v_{\max }\right)$. With multifrequency monitoring one can therefore test the theory by separating the changing flare component (coming from the evolving shock) and the constant baseline component (from the underlying jet, assumed to remain stable in shorter timescales). The conclusion was that as far as comparisons were possible, all the predictions of the $M \& G$ shocked jet model were in accordance with the data. 

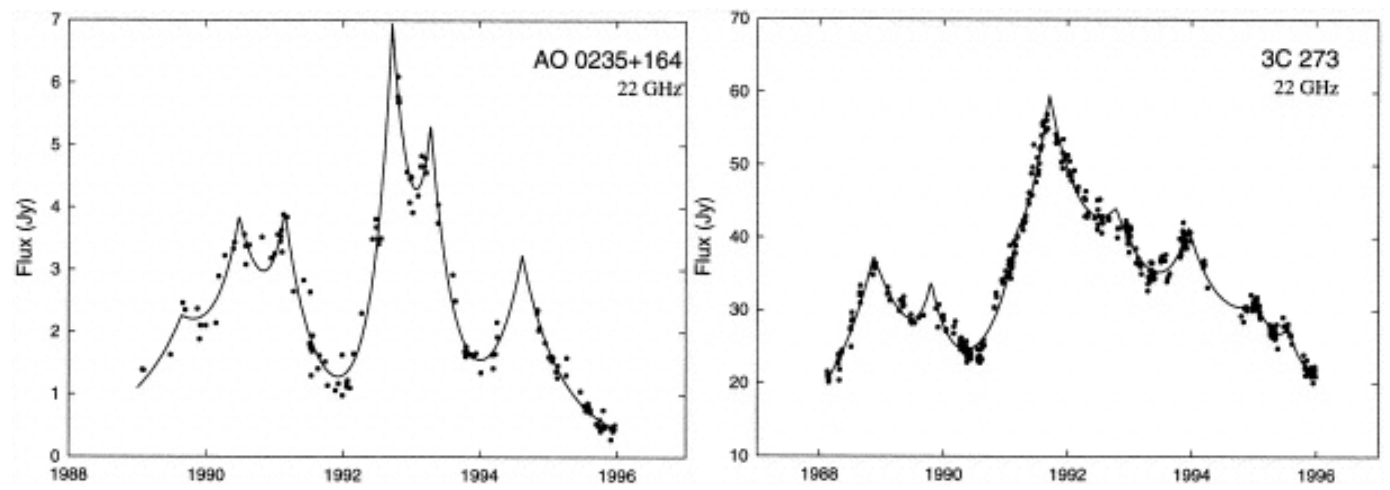

Figure 1. Two representative flux curves from Metsähovi monitoring program with exponential flare model fits. The observed flux (dots) is modelled as the sum of a constant baseline flux (not shown) and a number of exponential flares with the timescale and the peak flux of each flare as free parameters. Figure taken from [5].

Similar comparisons were later made using more extensive high-frequency data, in particular from the JCMT blazar monitoring program [6]. Combined with Metsähovi 22/37 GHz and University of Michigan cm-wavelength monitoring data (http://www.astro.lsa.umich.edu/obs/radiotel/umrao.php), this allowed a much better derivation of the shock spectrum and its evolution. Again, no significant differences could be found between observations and theory [7]. As far as we are aware, no observations have emerged since then which would be in direct conflict with the M\&G model predictions. The VLBI data also agree with the shocked jet scenario. The basic framework of synchrotron component modelling, growing and decaying relativistic shocks in a (relatively) stable jet thus seems to be secure.

During the more recent years two new tests of the shocked jet model have been done. Valtaoja et al. [5] found that all the major flux variations found in 85 sources included in the Metsähovi monitoring program could be very well described by a combination of a constant baseline flux and a series of similarly shaped exponential rises and decays, with the decay timescale 1.3 times the rise timescale (Figure 1). While this decomposition was purely mathematical modelling (in essence, a one-dimensional CLEAN procedure) with the aim to determine the fluxes, phases and peak epochs of the flares for a comparison with Compton gamma-ray data, the implication was clear: all the major total flux density variations have the same origin, identifiable with shocks in the otherwise stable jets. Later, Savolainen et al. [8] compared these exponential flares with VLBI monitoring data, demonstrating that the individual VLBI component flux curves were similar to the ones found from the total flux density flux curve decompositions.

A generalization to two dimensions, explicitly tied to shocks, was developed by Marc Türler, who created a code which uses as input the multifrequency monitoring set of all available data points $\mathrm{S}_{\mathrm{i}}\left(\mathrm{v}_{\mathrm{i}}, \mathrm{t}_{\mathrm{i}}\right)$, performing a simultaneous two-dimensional fit to the data. The code includes constraints from the Marscher and Gear shock scenario, using parameters which 


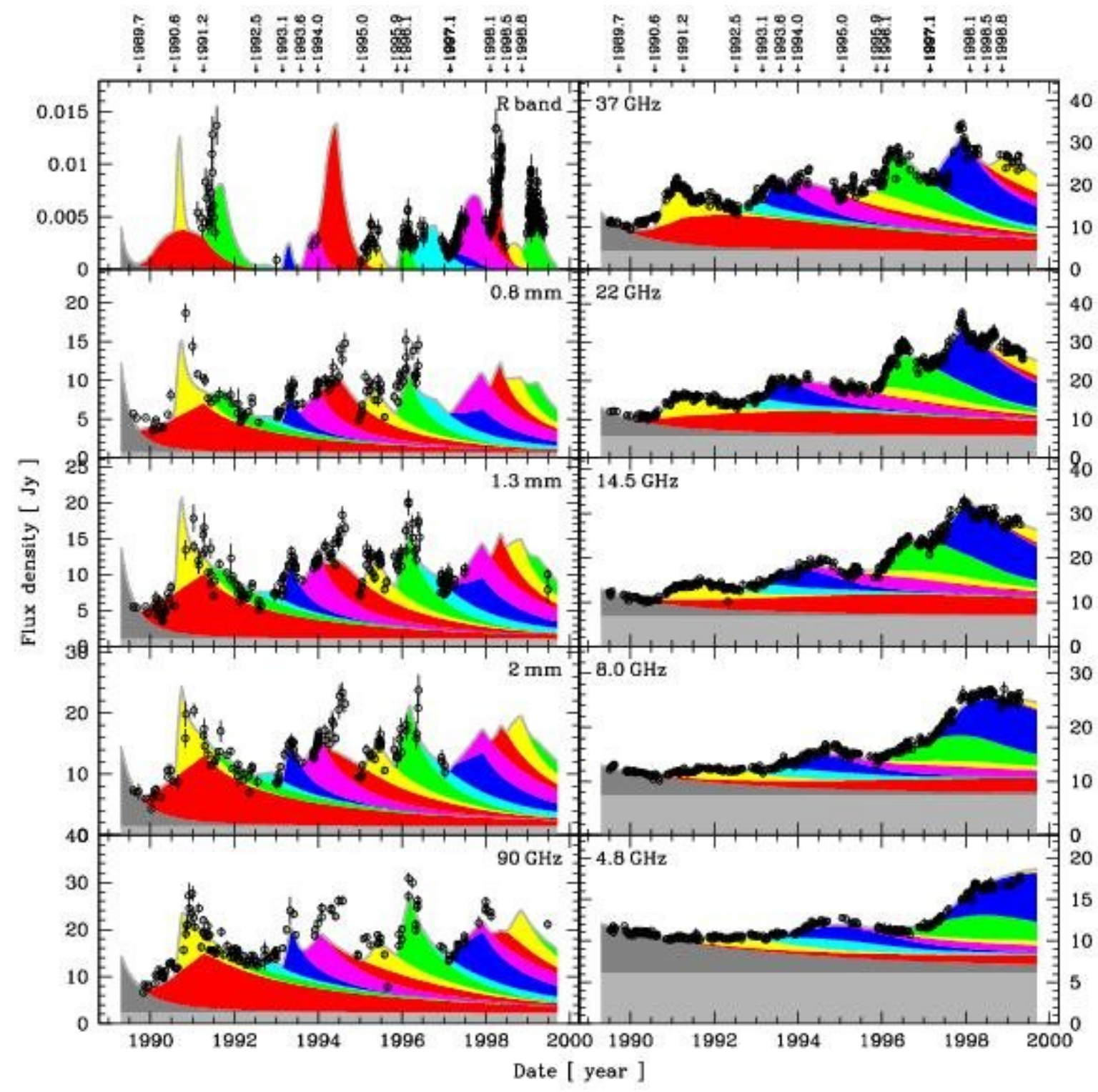

Figure 2. Ten years to radio-to-optical variations modelled by flares according to the code developed by Marc Türler and modified by Elina Lindfors. Figure taken from [10].

describe the evolution of the shock spectrum in the frequency-time regime according to the model description (see http//isdc.unige.ch/ turler/jets/). A modified form of the code was used by Lindfors et al. $[9,10]$ to model ten years of optical-to-radio variations in 3C 279. A very satisfactory fit with reasonable flow and shock parameters could be found (Figure 2). As far as we know, this is the so far most stringent test of the shocked jet model. The same code also describes galactic microquasars adequately, strongly suggesting that their flux variations are due to similar but scaled-down versions of shocked AGN jets [11, 12]. 


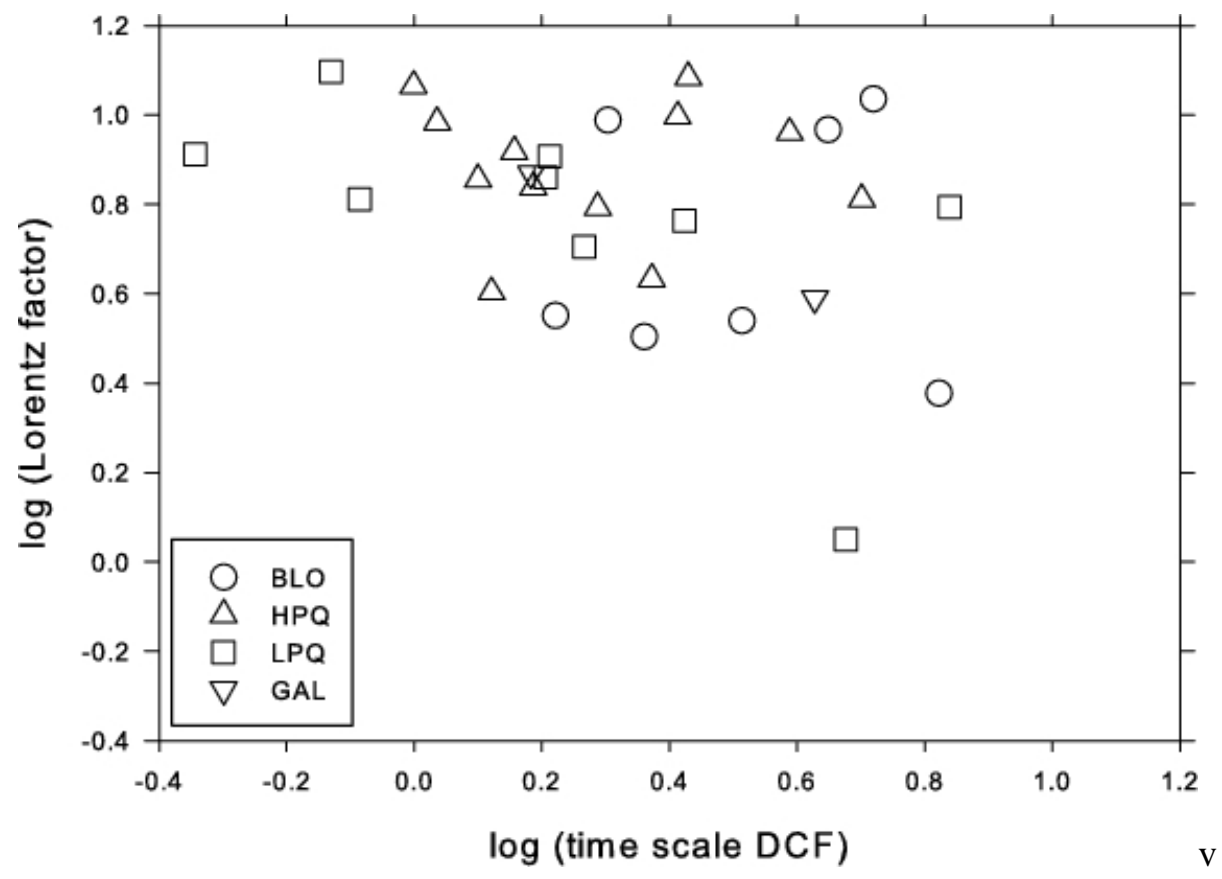

Figure 3. The typical time interval between radio flares (as estimated by the discrete correlation function timescale) versus the Lorentz factor of the flow. The fastest jets also have the most frequent outbursts. Figure taken from [13].

\subsection{Are all flux variations due to shocks?}

Does one mechanism really explain all flux variations? Although this seems to be the case with major flux variations, with timescales (in the millimeter regime) from weeks to years, it seems unlikely that minor variations are also bona fide shocks. Superposed on the major variations, they probably are rather described as flickering and instabilities in the shocks and their physics remain mainly unexplored. The well-known problems of intraday variability and too high intrinsic brightness temperatures (even if the IDV itself is external) are therefore not likely to be relevant to the shocked jet framework.

A more serious remaining question is the shape of the outbursts, in particular the extreme sharpness of the flare turnovers. This is a rule, not an exception, as Figure 1 shows. Except at lowest frequencies, where opacity and time delay effects dominate, one never sees a rounded flare with a gradually slowing flux increase, a plateau, and a slowly starting decreasing phase. Instead, the flares look like resonance peaks with a perfectly exponential rise abruptly turning into an equally perfectly exponential, slightly slower decrease. A look at Figure 2 shows also that the main failure of the M\&G-type description is its progressively growing inability to model the sharp peaks at higher frequencies. The hydrodynamic simulations, which at least qualitatively agree well with VLBI observations, also tend to produce a well-rounded evolution of shock spectra (e.g., [14]). Clearly, something fundamental is missing from our standard 
shock description. One might speculate that the missing part is also relevant for the IDV and other rapid flickering.

A major problem, from an observer's point of view, is the lack of shock models which both go beyond the simple description provided by the original $\mathrm{M} \& \mathrm{G}$ model and provide a possibility to compare the theory with observations. In particular, people doing numerical modelling should strive to extract basic data from their simulations: for example, how does the multifrequency flux change with time? Qualitative comparisons of numerical simulations with VLBI data are not enough; we also need quantitative comparisons.

Finally, we are almost totally in the dark regarding the underlying physics of the shocks. What are the primary causes and parameters which determine the specificity of the shocks we observe - their timescales, luminosities, speeds, frequencies, etc.? From the model fits of Türler and Lindfors we can clearly see that two subsequent shocks in the same source may have quite different characteristics. For example, one may peak at high frequencies and vanish before it reaches the low radio frequencies, while the other is still growing in the cm-regime. And how are shocks in various classes of sources different from each other? We know, for example, that the variability properties - that is, shock properties - are different in BL Lacs and blazar-type quasars, but what are the underlying differences in their physics? Acceleration, shock structure, jet structure, magnetic field configuration, energy budget, interactions, or what?

We have started to analyze the 30 years of Metsähovi data to search answers to some of these questions. One example of the work in progress is shown in Figure 3, taken from [13]. The interval between the flares - in other words, the basic activity timescale - seems to depend on the Lorentz factor of the jet. The faster the source, the shorter the duty cycle. One interesting possibility, not yet studied theoretically, is that the flare frequency is related to the width of the jet, since the opening angle is inversely correlated with the jet Lorentz factor [15], and the flaring frequency would thus be determined by internal jet instabilities instead of the activity of the black hole and accretion disk, external to the jet.

\section{Multifrequency comparisons, movies, and the origin of gamma radiation}

Radio variations are the only radiation component in blazars, for which we know their place of origin - shocks in the relativistic jet, rapidly advancing to distances of several parsecs downstream from the radio core, which in itself is at a quite large distance from the black hole and the accretion disk. Thus, even if one is not interested in the physics of shocks, total flux density variations may still be extremely useful since they provide a spatial and a temporal anchor to events occurring within AGN, a point source for optical or higher frequency observations. With monitoring data, we have a movie instead of just a snapshot. At the very least, we can state what was the state of the source during an observation; in the best case, we can find correlations between the total flux density and the other data, and thus say something about the origin of the observed radiation. For example, phenomena occurring after the beginning of the radio flare, or simultaneous to it, must be at a large distance from the black hole and the accretion disk.

An example of how knowledge of radio variations can illuminate a situation is shown in the recent paper of Marscher et al. [16], which represents the first clear localisation of radiation 
upstream of the radio core. The identification of the optical upstream flare depends on the observed polarization rotation $>180$ degrees, but it is also interestingly reflected in the comparison between optical and radio monitoring. The first optical flare, the one with the large polarization rotation, has no radio counterpart since it originates upstream of the radio core. As the shock emerges from the radio core, it produces a "standard" optical flare and a delayed radio flare. The picture is tantalizing: "orphan" optical flares with no radio counterparts come from upstream, optical flares with delayed radio flares from downstream.

\subsection{Origin of gamma radiation}

The origin of gamma radiation is an excellent example of the usefulness of total flux density radio monitoring. The Compton satellite revealed that many, possibly all, blazars emit copious amounts of gamma radiation at least intermittedly. The shape of the high frequency spectrum identifies it as inverse Compton radiation, photons scattered by the same relativistic electrons which produce the synchrotron component. But where do the photons come from, and where in the source is the IC component produced?

A large number of models have been fitted to the rather scarce and inaccurate Compton data. It is probably not uncharitable to say that the only thing that has been demonstrated by them is that virtually any model can produce a satisfactory fit to a single-epoch data set. To see this one needs only to look at the many papers in which the spectacular first Compton detection, that of 3C 279 in June 1991, has been modelled - with each paper using a different set of assumptions and parameter values. It is always possible to make up a story which appears to produce a satisfactory explanation to a single snapshot photograph! Indeed, only a very few modelling efforts have considered more than one epoch or one source, leaving the temporal aspects of gamma-ray emission unconstrained. Furthermore, the synchrotron spectrum that is used as the input for calculating the IC spectrum is almost always a theoretical construct, a onezone model in disagreement with both the epoch's actual data and with the basic shocked jet multicomponent framework. (As Figure 2 shows, at any given epoch there are always several shock components, any of which may in principle contribute significantly to the IC flux.)

The majority opinion is that in most blazars the seed photons are external to the jet, coming mainly from the accretion disk and the broad line region clouds. Synchrotron photons (internal to the jet) may also contribute, and for TeV sources SSC models are indeed the favored explanation. The most comprehensive modelling, incorporating seed photon contributions from the accretion disk, clouds, and the jet itself, has been done for the best-observed Compton source, 3C 279, by Hartman et al. [17]. The modelling included all the epochs for which the IC spectrum was obtained.

Multifrequency radio monitoring casts serious doubts over the external Compton scenarios. In order to work, the Hartman et al. and other EC models require that the site of gamma-ray emission is very close to the accretion disk, at the very least within the BLR clouds situated within a parsec from the black hole. Further out, the external photon field is too weak to provide a sufficient seed photon population. The EC models therefore provide a clear prediction of the order of events as a new disturbance propagates down the jet. First, there is an enhancement of 


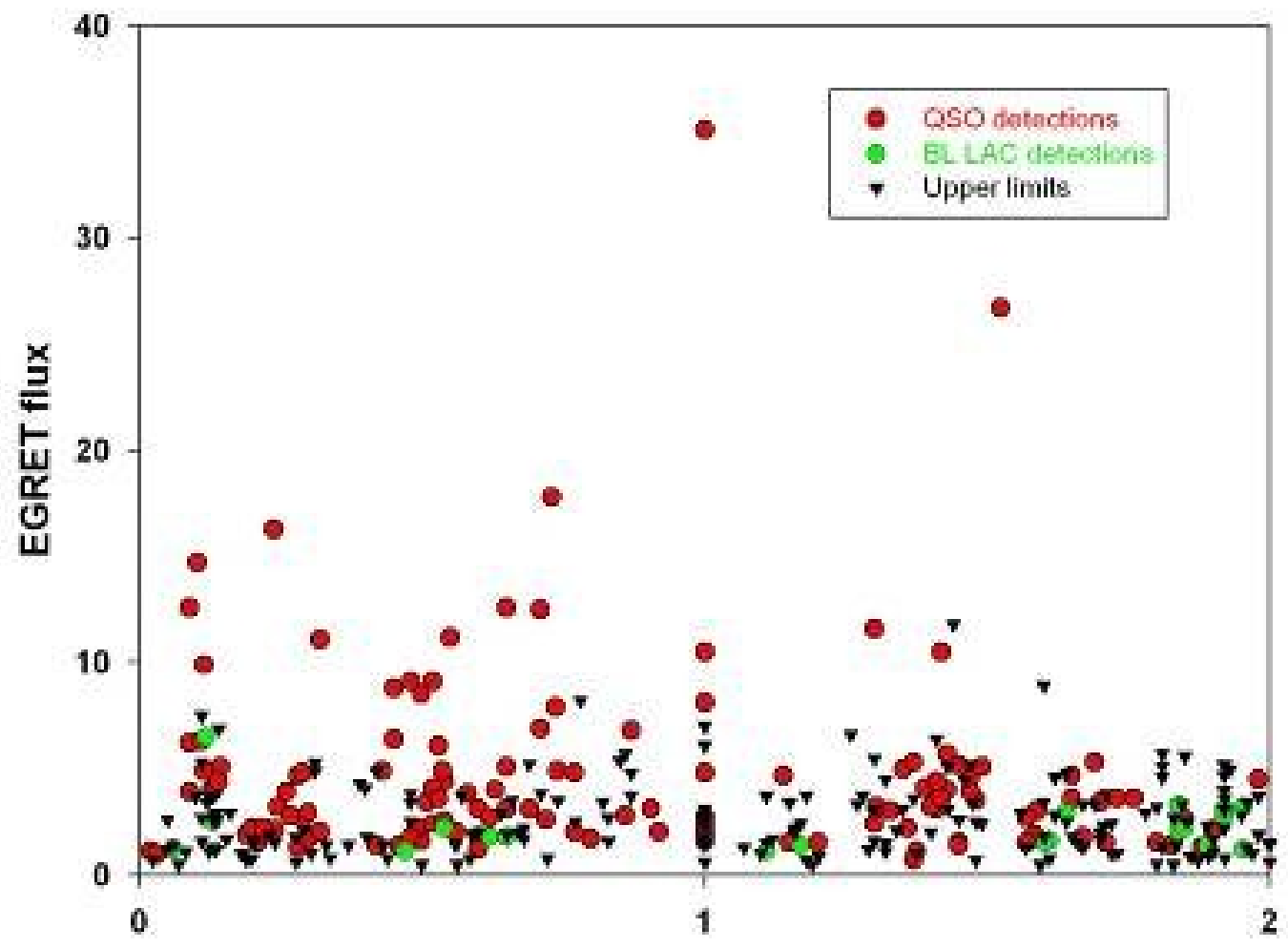

Flare phase $(0=$ start, $1=\max , 2=$ end $)$

Figure 4. The gamma-ray flux during Compton/EGRET pointings versus the $22 / 37 \mathrm{GHz}$ radio flare phase. All the pointings for which sufficient radio data was available are included. Almost all strong EGRET detections of quasars occurred during the rise or peak of a millimeter flare, strongly favoring a SSC origin. For EC origin, the detections should cluster around phases 0 and 2. (All the BL Lac detections were much weaker in comparison.) Figure taken from [18].

electrons - a new shock - emerging from the base of the jet, virtually touching the accretion disk. This creates the gamma-ray flare in the dense external photon field. Later, the disturbance propagates down the jet to larger distances where it becomes visible as a radio flare and a new VLBI component. The order of events is thus clear: first, a gamma-ray flare; then, the beginning of a radio flare and the emergence of a VLBI component from the radio core.

Yet the opposite is seen. Already with the first year's Compton data, Valtaoja and Teräsranta [19] demonstrated that gamma-ray emission is preferably seen during a radio flare, not before it starts. This correlation persists when all the Compton data and all the Metsähovi monitoring data is compared, as shown by Lähteenmäki and Valtaoja [18]. At 99.9\% confidence level, the millimeter flare has already started when a strong gamma-ray flare is seen by Compton (Figure 4). For weaker flares no such correlation is seen, perhaps indicating a different mechanism. Concentrating on 3C 279, Lindfors et al. [10] used the shock modelling described above and shown in Figure 2 to demonstrate that the more distant the dominant shock was from the radio core, the weaker was the gamma-ray flux measured during the ten Compton pointings. In each cased, the shock was already well beyond the BLR. The significance of the correlation was $99.98 \%$. Finally, comparing the Compton data with VLBI monitoring, Jorstad et al. [15] 
found that strong gamma-ray flares were on the average seen two months after the estimated zero separation epochs of the new VLBI components. The same average delay from the onset of the millimeter flare to the gamma-ray flare was also found by Lähteenmäki and Valtaoja. Not only the order of events but the timescale is also against an EC origin: two months in the observer's frame translates into a distance of several parsecs travelled by the shock downstream from the radio core before the gamma-rays are emitted, a distance well beyond the broad line region.

These statistical results, radio/gamma correlations anchoring the site of gamma emission far from the accretion disk, therefore strongly favor an SSC origin for the strongest gamma-ray flares seen by Compton. Although the chances of spurious correlations are small, we must wait for the GLAST data to be certain. There is also the small problem that SSC modelling has, almost without exception, failed to produce the observed amounts of gamma rays except using unphysical parameter values (with the noted exception of $\mathrm{TeV}$ sources). Our own SSC modelling, using not theoretical but real synchrotron multicomponent spectra derived from our multifrequency data, also fails by an order of magnitude to explain the 3C 279 gamma-ray fluxes [9].

The situation regarding the origin of gamma-ray emission is highly intriguing. On the one hand, comparison of all gamma-ray data with total intensity and VLBI monitoring gives strong statistical evidence against the EC models. On the other hand, SSC does not seem to work either. The new data from GLAST will, of course, settle the question; but whatever the outcome, real shock components must be used to model the high frequency data realistically, and the temporal framework cannot be neglected - and these data can only be obtained from multifrequency total intensity (and VLBI) monitoring.

\section{Fundamental physics of AGN}

As the final application of total flux density monitoring we consider the basic parameters of active galactic nuclei and their connections to observed data. It is easy to list the most fundamental parameters which must, somehow, shape the observed AGN activity: the black hole mass and spin, the accretion rate and the viewing angle. Secondary source parameters include the jet speed and the jet luminosity. These, and some others, must also somehow determine the parameter spaces of our various source classifications, such as BL Lac objects, blazars, non-blazar radio bright AGN, etc. How, for example, does the black hole mass influence the jet speed - do more massive AGN perhaps produce faster jets? What determines the jet luminosity? Are blazars ordinary radio-AGN seen at small viewing angles, as unification proposes? These, and other, fundamental questions can be explored with the help of total intensity radio variations.

Since the radio flares can be very nicely modelled by exponential flares, it is easy to calculate the associated apparent brightness temperature for each flare. These are usually found to exceed the theoretical limit for a synchrotron source, often by several orders of magnitude. The commonly accepted explanation is that the source (the shock) is in relativistic motion 


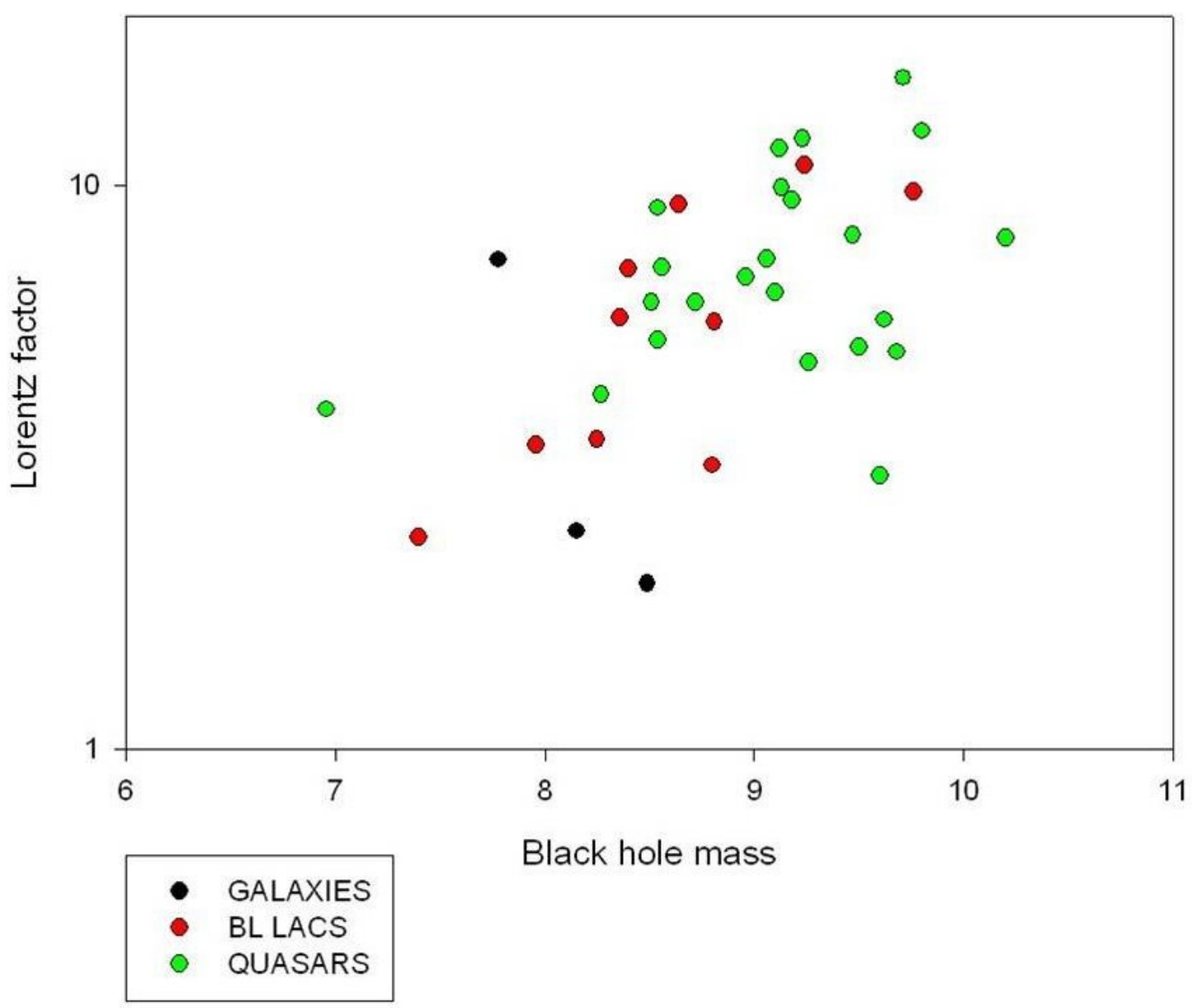

Figure 5. The estimated Lorentz versus black hole mass, using data from Lähteenmäki and Valtaoja [20] and mass determinations collected from the literature.

towards us, and therefore Doppler boosted with $D_{\text {var }}=\left[T_{b}(\mathrm{obs}) / \mathrm{T}_{\mathrm{b}}(\lim )\right]^{1 / 3}$. This method of derivng the Doppler factors for AGN was used by Lähteenmäki and Valtaoja [20] and, with a decade's worth of added monitoring data, by Hovatta et al. [21], who argued that the obtained values are much more accurate than the commonly used SSC- or gamma-derived ones.

\subsection{A blazar sequence?}

The Doppler boosting factors are useful for a variety of purposes. One unexpected finding is that (for unknown reasons) the Doppler factors have a very strong dependence on the peak frequency of the synchrotron spectrum [22]. The blazars with low peak frequencies have Doppler boosting factors up to 30, while the high-peaking BL Lacs and TeV sources are hardly Doppler-boosted at all. This flies in the face of TeV modelling, which usually assumes quite high Doppler boosting factors to obtain good fits with the observed gamma/TeV spectrum. It also demolishes the so-called blazar sequence, which is based on the assumption that all the sources have similar amounts of Doppler boosting and therefore the observed luminosities reflect true intrinsic luminosities. As recently shown in Nieppola et al. [22, 23], taking into 
account all the data and the effect of Doppler boosting, the claimed blazar sequence correlation vanishes. For BL Lacs, there may be an opposite correlation, with the TeV sources being the least Doppler-boosted BL Lacs, with the smallest Lorentz factors, and with the most powerful jets.

\subsection{Distributions and correlations}

If total intensity and VLBI monitoring data are joined, one can derive both the Lorentz factor $\Gamma$ and the jet viewing angle $\theta$ from $D_{\text {var }}$ and the apparent superluminal velocity $\beta_{\text {app }}$ using simple formulae [20]. One can then directly test the predictions of unification models and identify different $(\Gamma, \theta)$-distributions corresponding to different classes of objects. For example, BL Lacs are found to be different from quasars, having on the average smaller Lorentz factors, while blazar-type quasars (with high optical polarization) and ordinary quasars (with low optical polarization) have similar Lorentz factor distributions but blazar-type quasars have smaller viewing angles, just as unification predicts [20,21].

How does the black hole mass, arguably the single most fundamental parameter influencing AGN activity, correlate with the jet speed $\Gamma$, the Doppler-corrected synchrotron peak frequency $\cup_{\text {peak }}$, the Doppler-corrected peak luminosity $L_{\text {peak }}$, and the jet viewing angle $\theta$ ? We have initiated a program to find out, using our own variability Doppler factor estimates. the uniform data set of VLBI expansion speeds provided by the MOJAVE program and new black hole mass determinations from the literature and from our collaborators. In the GLAST era, two more parameters can soon be added to the correlation matrix: the peak frequency and the peak luminosity of the inverse Compton component. Progress is also being made in estimating the accretion rates of AGN, and spin determinations may also become possible in the future.

While all data is not yet available, preliminary analyses indicate that correlations do exist [24]. For example, the most massive black holes appear to produce the fastest jets, which also have the lowest synchrotron peak frequencies (Figure 5). When completed, the results of the analysis will provide basic input for theoretical and numerical modelling of AGN activity. The challenge will be to explain both the average trends (e.g., BH mass versus $\Gamma$ ) or lack of them, the differences between various classes of objects (e.g., $\mathrm{L}_{\text {peak }}(\mathrm{BL} \mathrm{Lac})$ vs $\left.\mathrm{L}_{\text {peak }}(\mathrm{QSO})\right)$, and the reasons of individual deviations from average behavior typical for similarly classified objects. Only when we can derive the observed parameters from the fundamental physical parameters can we say that we truly are starting to understand the AGN phenomenon.

\section{Conclusions}

We have illustrated the usefulness of total flux density monitoring with some examples drawn from previous and current research at Metsähovi and Tuorla observatories. While monitoring is a very time-consuming effort, there are things which simply cannot be done in any other way: however detailed, a snapshot can never replace a movie. Radio monitoring can function as both a spatial and a temporal anchor for any observations. Monitoring also provides statistics, both for a single source and for a sample of sources. With the limited nature of data we have for synchrotron-emitting sources, a large number of different theoretical constructs 
may explain any single data set, such as a single-epoch spectrum, but statistical analysis of all available data can often produce clear-cut tests of models.

Especially when combined with other multifrequency and multiapproach data, radio monitoring is a very powerful tool for testing various theoretical models, AGN classification and unification, and a key for studying the basic physics of jets.

\section{References}

[1] R. Blandford \& A. Königl, Relativistic jets as compact radio sources, ApJ 232, 34 (1979)

[2] A. Marscher \& W. Gear, Models for high-frequency radio outbursts in extragalactic sources, with application to the early 1983 mlilimeter-to-infrared flare of 3C 273, ApJ 298, 114 (1985)

[3] E. Valtaoja et al., Five years monitorimg of extragalactic radio sources. II. Quiescent spectra and the evolution of outbursts, A\&A 203, 1 (1988)

[4] E. Valtaoja et al., Five years monitoring of extragalactic radio sources. III. Generalized shock models and the dependence of variability on frequency, A\&A 254, 71 (1992)

[5] E. Valtaoja et al., Total flux density variations in extragalactic radio sources, I. Decomposition of variations into exponential flares, ApJS 120, 95 (1999)

[6] J. Stevens et al., Multifrequency observations of blazars. 5. Long-term millimeter, submillimeter, and infrared monitoring, ApJ 437, 91 (1994)

[7] J. Stevens et al., The spectral evolution of high-frequency radio outbursts in the blazar PKS 0420O14, MNRAS 275, 1146 (1995)

[8] T. Savolainen et al., Connections between millimetre continuum variations and VLBI structure of 27 $A G N, A \& A$ 394, 851 (2002)

[9] E. Lindfors et al., SSC mechanism in the gamma-ray blazar 3C 279, A\&A 440, 845 (2005)

[10] E. Lindfors et al., Synchrotron flaring in the jet of $3 C$ 279, A\&A 456, 895 (2006)

[11] M. Türler et al., Quasar jet emission model applied to microquasar GRS 1915+105, A\&A 415, 35 (2004)

[12] E. Lindfors et al., Synchrotron flaring behaviour of Cygnus X-3 during the February-March 1994 and September 2001 outbursts, A\&A 473, 923 (2007)

[13] T. Hovatta et al., Statistical analyses of long-term variability of AGN at high radio frequencies, A\&A 469, 899 (2007)

[14] J. Gomez et al., Hydrodynamical models of superluminal sources, ApJ 482, 33 (1997)

[15] S. Jorstad et al., Multiepoch Very Long Baseline Array observations of EGRET-detected quasars and BL Lacertae objects: superluminal motion of gamma-ray bright blazars, APJS 134, 181 (2001)

[]16] A. Marscher et al., The inner jet of an active galactic nucleus as revealed by a radio-to- $\gamma$ outburst, Nature 452, 966 (2008)

[17] R. Hartman et al., Multiepoch multiwavelength spectra and models for blazar 3C 279, ApJ 553, 683 (2001) 
[18] A. Lähteenmäki \& E. Valtaoja, Testing inverse Compton models for active galactic nuclei with gamma-ray and radio observations, ApJ 590, 95 (2003)

[19] E. Valtaoja \& H. Teräsranta, Gamma radiation from radio shocks in AGN jets, A\&A 297, 13 (1995)

[20] A. Lähteenmäki \& E. Valtaoja, Total flux density variations in extragalactic radio sources. III. Doppler boosting factors, Lorentz factors, and viewing angles for active galactic nuclei, ApJ 521, 493 (1999)

[21] T. Hovatta et al., in preparation (2008)

[22] E. Nieppola et al., arXiv0803.0654N (2008)

[23] E. Nieppola et al., Spectral energy distributions of a large sample of BL Lacertae objects, A\&A 445, 441 (2006)

[24] E. Valtaoja et al., Hydrodynamics of small-scale jets: obsrvational aspects, in ASP Conf. Ser. 386, 388 (2008) 